OPEN ACCESS

Edited by: Madhuchhanda Bhattacharjee, University of Hyderabad, India

Reviewed by: Inyoung Kim, Virginia Tech, United States Nitish Kumar Mishra, University of Nebraska Medical Center, United States

*Correspondence:

Sajida Perveen

Sajida.uaar@gmail.com

Specialty section: This article was submitted to Bioinformatics and Computational Biology, a section of the journal Frontiers in Genetics

Received: 08 July 2019 Accepted: 09 October 2019 Published: 07 January 2020

Citation:

Perveen S, Shahbaz M, Ansari MS, Keshavjee K and Guergachi A (2020) A Hybrid Approach for Modeling Type 2 Diabetes Mellitus Progression.

Front. Genet. 10:1076. doi: 10.3389/fgene.2019.01076

\section{A Hybrid Approach for Modeling Type 2 Diabetes Mellitus Progression}

\author{
Sajida Perveen ${ }^{1 *}$, Muhammad Shahbaz ${ }^{1,2}$, Muhammad Sajjad Ansari ${ }^{3}$, \\ Karim Keshavjee ${ }^{2,4}$ and Aziz Guergachi ${ }^{2,5,6}$
}

${ }^{1}$ Department of Computer Science \& Engineering, University of Engineering \& Technology, Lahore, Pakistan, ${ }^{2}$ Research Lab for Advanced System Modelling, Ryerson University, Toronto, ON, Canada, ${ }^{3}$ Division of Science and Technology, University of Education, Lahore, Pakistan, 4 Institute for Health Policy, Management and Evaluation, University of Toronto, Toronto, ON, Canada, 5 Ted Rogers School of Information Technology Management, Ryerson University, Toronto, ON, Canada,

${ }^{6}$ Department of Mathematics \& Statistics, York University, Toronto, ON, Canada

Type 2 Diabetes Mellitus (T2DM) is a chronic, progressive metabolic disorder characterized by hyperglycemia resulting from abnormalities in insulin secretion, insulin action, or both. It is associated with an increased risk of developing vascular complication of micro as well as macro nature. Because of its inconspicuous and heterogeneous character, the management of T2DM is very complex. Modeling physiological processes over time demonstrating the patient's evolving health condition is imperative to comprehending the patient's current status of health, projecting its likely dynamics and assessing the requisite care and treatment measures in future. Hidden Markov Model $(H M M)$ is an effective approach for such prognostic modeling. However, the nature of the clinical setting, together with the format of the Electronic Medical Records (EMRs) data, in particular the sparse and irregularly sampled clinical data which is well understood to present significant challenges, has confounded standard HMM. In the present study, we proposed an approximation technique based on Newton's Divided Difference Method (NDDM) as a component with HMM to determine the risk of developing diabetes in an individual over different time horizons using irregular and sparsely sampled EMRs data. The proposed method is capable of exploiting available sequences of clinical measurements obtained from a longitudinal sample of patients for effective imputation and improved prediction performance. Furthermore, results demonstrated that the discrimination capability of our proposed method, in prognosticating diabetes risk, is superior to the standard HMM.

Keywords: type 2 diabetes mellitus, machine learning, hidden Markov model, prognostic modelling, risk prediction, risk scoring

\section{INTRODUCTION}

Diabetes mellitus is a metabolic disorder of multiple etiologies (Alberti and Zimmet, 1998). It can lead to progressive development of multidimensional complications as to vascular system of human body (Einarson et al., 2018). Complications of micro-vascular endpoints may include retinopathy, nephropathy and neuropathy, while the ones related to macro-vascular endpoints may include 
macro-vascular endpoints may include stroke, peripheral vascular disease and ischemic cardio vascular disease (McEwen and Herman, 2017; Zou et al., 2018). Diabetes mellitus is found to be potentially an independent contributing factor for premature mortality and reduced life expectancy (Atlas, 2015). There is significant evidence that the prevalence of diabetes mellitus is rising shockingly at a faster pace affecting middleaged adult population disproportionately (Emerging Risk Factors Collaboration, 2010). Globally, about 382 million people were diagnosed with diabetes in 2013 -bringing $6.6 \%$ of the world's population under this disease (Perveen et al., 2016). Studies indicate it is likely to escalate by $51 \%$ by 2030 (Wild et al., 2004).

Diabetes accounts for a considerable proportion of healthcare resources worldwide (Liebl et al., 2000; Perveen et al., 2018a). Even though a century after the invention of insulin, diabetes still calls for significant therapeutic measures. Degenerative complications (like renal failure and cardio vascular disease) in a substantial fraction of diabetic patients are the reasons behind it to some extent (Pambianco et al., 2006; Gregg et al., 2014). Health-care cost for diabetic patients is anticipated to be about $\$ 490$ billion for 2030, which accounts for $11.6 \%$ of global health spending (Perveen et al., 2016).

Several pathogenic processes are believed to play long-winded role in the development process of diabetes (Perveen et al., 2018a). Diabetes does not manifest noticeable symptoms at the initial stage of its development (Ramachandran, 2014). Rather, it demonstrates a highly covert nature of symptoms particularly at the early stage of disease until it substantially developed and evident symptoms transpire (American Diabetes Association, 2014). Due to this asymptomatic intricacy of diabetes, the identification a-priori of pre-diabetic individuals remains quite challenging.

In 2002, Diabetes Prevention Program (DPP) demonstrated that lifestyle intervention directed at exercise and reducing weight was more effective to reduce or delay the risk of developing Type 2 Diabetes Mellitus (T2DM) than the treatment with Metformin (Knowler et al., 2002; Lindstrom et al., 2003; Li et al., 2008). Several meta-analysis and clinical trial also suggests that early interventions can delay or altogether counteract the developing mechanism of diabetes mellitus ( $\mathrm{Li}$ et al., 2008). However, the constraints and cost of these interventions for individuals are primary the arguments against their provision. Furthermore, the interventions can be cost effective only when appropriate target population is used i.e. the one which has a high likelihood of developing diabetes at the baseline (Diabetes Prevention Program Research Group, 2003).

Within this context, the focus of disease management needs to be changed as follows; from hazard to vulnerability reduction; from reactive to proactive; from response management to risk management. However, these changes require novel technological solutions with an emphasis on management of early stages of the disease.

There are various well know diabetes risk prediction model, including FDRSM (Wilson et al., 2007), ARIC (Kahn et al., 2009), San-Antonio (Stern et al., 2002), AUSDRISK (Chen et al., 2010) and FINDRISC (Lindström and Tuomilehto, 2003) that provide the opportunity to estimate the risk of developing diabetes. However, the selection of appropriate risk scoring model is a cumbersome and challenging process (Mashayekhi et al., 2015; Perveen et al., 2016). In general these scoring models are based on prospective studies (like Framingham heart study (Wilson et al., 2007) that prove to be very expensive and also time consuming. Furthermore, these risk scoring models also inherit bias due to differential loss to follow up along with a progressive time taking and costly screening procedure that again makes the intervention measures ineffective and impractical.

Machine Learning (ML) techniques, over the last few years, have been seen to exhibit an increased relevance to a variety of objectives, including risk assessment (Perveen et al., 2018b). This rich knowledge may be useful for some decisive steps to characterize disease risk and progression. ML techniques seem to be an appealing option for the prevention of T2DM. In this context, Electronic Medical Records (EMRs) create a promising horizon for establishing rich and complex physiological models (Gunter and Terry, 2005; Liu et al., 2015). Hence, it is a driving factor for the adoption of state of art data-driven techniques, bringing together the opportunities to automate health-care related tasks (Birkhead et al., 2015).

Hidden Markov Model (HMM) has been extended to deal with the sequential data (Lai et al., 2016). It is particularly an effective approach to predict the future risk of a disease in an individual using sequences of clinical measurements obtained from longitudinal samples of patients (El Nahas et al., 2012; Srikanth, 2015). While classical HMM is used for disease progression modeling, in general, it is not suitable because it assumes that measurement data is collected regularly at discrete time intervals (Liu et al., 2015). However, in reality patient visits are often irregular in time, as a consequence of scheduling issues, selectively miss some pre-scheduled visits or be assessed at self-selected points in time and changes in symptomatology (i.e. patients may visit more often when unwell or vice versa). Consequently, yield electronic medical records with observations sequence irregularly or sparsely sampled and grossly violate the model assumption. Furthermore, these effects also make learning and inference problems more complicated.

In order to resolve the above-mentioned problem and to provide a prompt and comprehensive analysis of EMRs data in the present study, we propose to use HMM with a formulation approach based on Newton's Divided Difference Method (NDDM) to develop a simple and robust tool to investigate the future diabetes incidents by learning dynamic interactions from longitudinal data. The early identification of pre-diabetic individuals, even when they are in a normoglycemic state, provides further reason for targeting interventions in those, most likely to benefit. Furthermore, the utility of the proposed formulation approach in conjunction with standard HMM has not been explored to address the problem of sparse and irregularly sampled EMRs data which is an unavoidable issue in almost every health-care dataset. 


\section{MATERIALS AND METHODS}

\section{Study Design, Participants and Data Collection}

This prospective study primarily focuses on EMRs data obtained from the Canadian Primary Care Sentinel Surveillance Network (CPCSSN) (http://cpcssn.ca/). This prospective dataset contains 812,007 records of 172,168 unique individuals, those were enrolled in CPCSSN between the years 2003 and 2015 .

Temporal feature vectors of clinical measurements for each patient was generated based on the patient's extracted EMRs data from the observation window. Each feature vector representation includes information related to $\mathrm{BP}$ (Blood Pressure), sex, Body Mass Index (BMI), Fasting Blood Glucose (FBG) levels, age, High Density Lipoprotein (HDL), Light Density Lipoprotein (LDL), Glycated Hemoglobin (HbA (1c), total cholesterol and Triglycerides (TG). All patients were assigned a unique ID to track the health status during the follow-up period. In this article we intended to explore the potential of EMRs data to assess T2DM risk, thus we did not add any other outside covariate (i.e. physical activities. To capture a representative cohort, all individuals who have at least 5 visits with 1 year time interval gap till the end of 8 years of follow-up and have information for all the attributes included in this proposed study, as mentioned in Table 2, were eligible for inclusion. Approximately 170,250 patients out of 172,168 do not meet minimum inclusion criteria and excluded from the research sample. Thus, this prospective study resulted in a total of 1981 participants for final dataset. In terms of patient demographics, the average age was 40 years, range between 18 to 83 years.

Generally, early risk identification of T2DM in populations is appropriate when it will be an organized continuous process rather than a single time and isolated effort. Furthermore, there should be a reasonable balance in the costs of case identification and treatment in relation to healthcare cost as a whole. Therefore, using these sequences of clinical measurements extracted from CPCSSN a set of experiments was completed to measure how well the proposed data-driven and multivariate predictive model performs in evaluating the ongoing risk of T2DM over varied length of prediction windows.

The primary outcome of interest is to prognosticate risk of developing diabetes in an individual over a series of 8 time horizons: 1 year to 8 years. Such modeling confers an epistemic and instrumental value that manifests in the ability to take intervention measures on time and/or provide individualized treatments based on disease risk (Yoon et al., 2016). By having follow-up through 2015, we ensured that all individuals had at least 5 years of follow-up regardless of disease status.

\section{Proposed Method}

Considering the objective of the proposed research and the above mentioned challenges the proposed method consists of two main components. (1) Handling sparse and irregularly sampled time series EMRs data and (2) the development of prognostic prediction model based on HMM using relevant risk factors for prognostic prediction of diabetes risk over different time horizons.

Handling Sparse and Irregularly Sampled EMRs Data Modeling the clinical condition of a particular patient using evidential physiological data is a ubiquitous problem that arises in many healthcare settings (Liu et al., 2015; Schulam and Saria, 2015; Yoon et al., 2016; Hoiles and Van Der Schaar, 2016). In this context, EMRs data is one of the fundamental resources to derive medical insights and/or support medical practice However, management and processing of such data is challenging due to various factors that are inherent in the data itself.

In particular, the dynamic range of the time scale in EMRs is one of the bothersome characteristics of EMRs data and potentially the contributing factor for sparse and irregularly sampled clinical data. Irregularly sampled (or non-uniformly sampled) time series are characterized by variable time intervals between successive observations ( $\mathrm{Li}$ and Marlin, 2016). When the intervals between successive observations are long, the time series are said to be sparsely sampled. Irregularity is caused by the fact that patients will only have EMRs data recorded when they visit the hospital.

Consider a longitudinal EMRs data of $\mathrm{n}$ independent time series $D=\left\{S_{1}, S_{2}, \ldots ., S_{n}\right\}$ recorded at a specific time, for instance, hours, months, or years. Each $S_{i}$ is represented as a list of time points $t_{i}=\left\{t_{i 1}, t_{i 2}, \ldots ., t_{i}\left|S_{i}\right|\right\}^{T}$, and a list of corresponding values, $\mathrm{y}_{\mathrm{i}}=\left\{\mathrm{y}_{\mathrm{i} 1}, \mathrm{y}_{\mathrm{i} 2}, \ldots \ldots, \mathrm{y}_{\mathrm{i}}\left|\mathrm{S}_{\mathrm{i}}\right|\right\}^{\mathrm{T}}$. We assume that each time series is defined over a regular time interval $[0, \mathrm{~T}]$. However, for irregularly sampled time series we do not assume that all of the time series are defined on the same collection of time points (i.e., $t_{i} \neq t_{j}$ in general), we do not assume that the intervals between time points are uniform. We also do not assume that the number of observations in different time series is the same (i.e., $\left|S_{i}\right| \neq\left|S_{j}\right|$ in general) and evolves smoothly over time. In other words longitudinal record of each patient is considered as a sparse matrix with features and a time dimension.

CPCSSN data is prone to sparsity and irregularity and tended to violate the HMM based prognostic model assumption to some degree. In addition, sparse and irregularly sampled time series data is itself different from traditional structured data to fit a model. Therefore before developing analytics solution from such data for prognostic prediction of T2DM risk over different time horizons, we propose to use an approximation approach based on NDDM (Kalu, 2009). Which approximates the values for those unknown observations in the longitudinal matrix for each patient by exploring the latent structures on both feature and time dimensions from the information which becomes available from relevant observations in EMRs.

Furthermore, it would be worth exploring whether the proposed method could affect the classification accuracy of the prediction model. However, according to our best knowledge this is the first study that incorporated NDDM to handle the problem of sparse and irregularly sampled EMRs data before developing numerical solution for prognostic prediction of diabetes risk over different time horizons. 
NDDM is a standard method used for interpolating polynomial in terms of divided differences. The interpolation problem can be defined as follows: given a set of pairs of numbers $\left(\mathbf{x}_{\mathbf{0}}, \mathbf{f}_{\mathbf{0}}\right),\left(\mathbf{x}_{1}, \mathbf{x}_{1}\right), \ldots \ldots . .,\left(\mathbf{x}_{\mathbf{n}}, \mathbf{f}_{\mathbf{n}}\right)$, with all $\mathbf{x}_{1}, \mathbf{x}_{2}, \ldots ., \mathbf{x}_{\mathbf{n}}$ are different and not necessarily equally spaced, whereas $\mathbf{f}_{\mathbf{i}}$ may be the value of some mathematical function $\mathbf{f}(\mathbf{x})$ or empirically obtained in an experiment or observation. The interpolation problem is to find a polynomial $\mathbf{P}_{\mathbf{n}}(\mathbf{x})$ such that $\mathbf{P}_{\mathbf{n}}(\mathbf{x})=\mathbf{f}_{\mathbf{0}}, \mathbf{P}_{\mathbf{n}}$ $\left(\mathbf{x}_{1}\right)=\mathbf{f}_{1}, \ldots, \mathbf{P}_{\mathbf{n}}\left(\mathbf{x}_{\mathbf{n}}\right)=\mathbf{f}_{\mathbf{n}}$.

The polynomial $P_{n}(x)$ is used to estimate value for all $x$ such that $P_{n}(x)$ is approximately $f(x)$ or to get values for $x_{s}$ at which no measurement was taken. This interpolation polynomial can be written in the Newton form as follows (Mathews, 1986):

$$
\begin{aligned}
\mathrm{P}_{\mathrm{n}}(\mathrm{x})= & \mathrm{f}\left[\mathrm{x}_{0}\right]+\mathrm{f}\left[\mathrm{x}_{0}, \mathrm{x}_{1}\right]\left(\mathrm{x}-\mathrm{x}_{0}\right)+\mathrm{f}\left[\mathrm{x}_{0}, \mathrm{x}_{1}, \mathrm{x}_{2}\right]\left(\mathrm{x}-\mathrm{x}_{0}\right)\left(\mathrm{x}-\mathrm{x}_{1}\right) \\
& +\mathrm{f}\left[\mathrm{x}_{0}, \mathrm{x}_{1}, \mathrm{x}_{2} \mathrm{x}_{3}\right]\left(\mathrm{x}-\mathrm{x}_{0}\right)\left(\mathrm{x}-\mathrm{x}_{1}\right)\left(\mathrm{x}-\mathrm{x}_{2}\right)+\ldots \ldots \\
& +\mathrm{f}\left[\mathrm{x}_{0}, \mathrm{x}_{1}, \mathrm{x}_{2}, \ldots \mathrm{x}_{\mathrm{n}}\right]\left(\mathrm{x}-\mathrm{x}_{0}\right)\left(\mathrm{x}-\mathrm{x}_{1}\right)\left(\mathrm{x}-\mathrm{x}_{2}\right) \ldots \ldots\left(\mathrm{x}-\mathrm{x}_{\mathrm{n}}\right)
\end{aligned}
$$

Where $\left\{f\left[\mathrm{x}_{0}\right], \mathrm{f}\left[\mathrm{x}_{0}, \mathrm{x}_{1}\right]\right.$ and $\left.\mathrm{f}\left[\mathrm{x}_{0}, \mathrm{x}_{1}, \mathrm{x}_{2}\right]\right\}$ are finite divided differences and $\mathrm{f}\left[\mathrm{x}_{0}\right], \mathrm{f}\left[\mathrm{x}_{0}, \mathrm{x}_{1}\right]$ and $\mathrm{f}\left[\mathrm{x}_{0}, \mathrm{x}_{1}, \mathrm{x}_{2}\right]$ are the first, second, and third order finite divided differences, respectively that can be defined as below:

$$
\begin{gathered}
f\left[x_{0}\right]=f\left(x_{0}\right) \\
f\left[x_{0}, x_{1}\right]=\frac{f\left(x_{1}\right)-f\left(x_{0}\right)}{x_{1}-x_{0}} \\
f\left[x_{0}, x_{1}, x_{2}\right]=\frac{\frac{f\left(x_{2}\right)-f\left(x_{1}\right)}{x_{1}-x_{0}}-\frac{f\left(x_{1}\right)-f\left(x_{0}\right)}{x_{1}-x_{0}}}{x_{2}-x_{0}}
\end{gathered}
$$

Similarly, $\mathrm{n}^{\text {th }}$ Divided Difference is given by

$f\left[x_{i}, x_{i+1}, x_{i+2}, \ldots, x_{i+n}\right]=\frac{f\left[x_{i}, x_{i+1}, x_{i+2}, \ldots, x_{i+n}\right]-f\left[x_{i}, x_{i+1}, x_{i+2}, \ldots, x_{i+n-1}\right]}{x_{i+n}-x_{i}}$

\section{Prognostic Modeling}

Once the dataset is prepared by taking the output of the proposed approximation method the next crucial task is potentially contributing risk factors selection. Therefore, to optimally select the potentially contributing factors, Logistic Regression (LR) analysis is performed on the derived dataset that consist of risk factors related to demographics, vitals, diagnoses and laboratory tests results as given in Table $\mathbf{1}$.

Models were trained and evaluated using only risk factors that exhibit significant relationship with T2DM when LR analysis was performed. Models were trained and evaluated using only risk factors that exhibit significant relationship with T2DM when LR analysis was performed. Subsequently, the parameters are drawn

\begin{tabular}{|c|c|}
\hline Predictors & Findings \\
\hline \multicolumn{2}{|l|}{ Demographic (Sex, Age) } \\
\hline Female, sample size (\%) & $100,566(57)$ \\
\hline Male age mean $\pm S D, Y r$ & $48.2 \pm 24.1$ \\
\hline Female age mean $\pm \mathrm{SD}, \mathrm{Yr}$ & $49.5 \pm 24.8$ \\
\hline Male age mean $\pm \mathrm{SD}, \mathrm{Yr}$ & $48.2 \pm 24.1$ \\
\hline \multicolumn{2}{|l|}{ Vital Signs/clinical measures } \\
\hline Systolic BP, mean $\pm \mathrm{SD}, \mathrm{mm} \mathrm{Hg}$ & $129.34 \pm 17.183$ \\
\hline Chronic obstructive pulmonary disease frequency (\%) & $9,939(2.4)$ \\
\hline Dementia frequency (\%) & $12,007(1.8)$ \\
\hline Depression frequency (\%) & $32,672(10)$ \\
\hline Diabetes Mellitus frequency (\%) & $26,077(6)$ \\
\hline Epilepsy frequency (\%) & $5,553(0.8)$ \\
\hline Hypertension frequency (\%) & $61,370(13)$ \\
\hline Osteoarthritis frequency (\%) & $37,274(7)$ \\
\hline Parkinson's Disease frequency (\%) & $1,825(0.2)$ \\
\hline \multicolumn{2}{|l|}{ Lab Values } \\
\hline Fasting blood glucose, mean $\pm \mathrm{SD}, \mathrm{mmol} / \mathrm{L}$ & $5.54 \pm 1.91$ \\
\hline $\mathrm{TG}$, mean $\pm \mathrm{SD}, \mathrm{mmol} / \mathrm{L}$ & $1.523 \pm 0.962$ \\
\hline $\mathrm{LDL}$, mean $\pm \mathrm{SD}, \mathrm{mmol} / \mathrm{L}$ & $2.83 \pm 0.99$ \\
\hline High density lipoprotein, mean $\pm \mathrm{SD}, \mathrm{mmol} / \mathrm{L}$ & $1.3893 \pm 0.416$ \\
\hline $\mathrm{BMl}$, mean $\pm \mathrm{SD}, \mathrm{kg} / \mathrm{m}^{2}$ & $37.113 \pm 1528.71$ \\
\hline $\mathrm{HbA}(1 \mathrm{c})$, mean $\pm \mathrm{SD}, \mathrm{mmol} / \mathrm{L}$ & $6.268 \pm 0.976$ \\
\hline Cholesterol mean $\pm \mathrm{SD}, \mathrm{mmol} / \mathrm{L}$ & $4.893 \pm 1.159$ \\
\hline
\end{tabular}
from training dataset using Baum-Welch algorithm. However, to fit the predictive model, we used standard GaussianHMM, a variant of classical HMM. It is a finite probability density distribution model that has been widely deployed as temporal
TABLE 1 | Characteristics of the population in the CPCSSN database.

$S D$, standard deviation; $Y r$, year; $B P$, blood pressure; $L D L$, light density lipoprotein; $H b A(1 c)$, glycated hemoglobin; TG, triglycerides; BMI, body mass index; HDL.

* Some patients have more than 1 disease in the database.

latent variable model for modeling dynamic systems (Kenny et al., 1990; Artières et al., 2000). Several variants of the basic hidden markov model have been proposed, with slightly different functionality (Rabiner, 1989). The basic concept was published in a series of classic papers by Baum and Petrie (Baum and Petrie, 1966). As our data retained continuous variables thus, the observation probability assumes the Gaussian distribution. Our model has structural assumptions about the underlying structure of the process and assumed to be composed of the set of hidden states $S=\left\{s_{1}, s_{2}, s_{3} \ldots . . s_{m}\right\}$ (corresponding to diabetic or nondiabetic in our case) in the model, initial state distribution, an observational symbol distribution (e.g. Gaussian) of each state and a state transition matrix generally parameterized by a set probabilities used for further analysis, as follows:

$$
\begin{gathered}
\theta=(\pi=\underbrace{\pi_{\mathrm{i}}=\left\{\mathrm{q}_{1}=\mathrm{s}_{\mathrm{i}}\right\}}_{\text {Prior probability, }} \quad \mathrm{A}=\underbrace{\mathrm{a}_{\mathrm{i}, \mathrm{j}}=\mathrm{p}\left(\mathrm{q}_{\mathrm{t}+1}=\mathrm{s}_{\mathrm{j}} \mid \mathrm{q}_{\mathrm{t}}=\mathrm{s}_{\mathrm{i}}\right)}_{\text {Transition propabilities matrix }} \\
\mathrm{B}=\underbrace{\mathrm{b}_{\mathrm{i}}(\mathrm{K})=\mathrm{b}_{\mathrm{i}}\left(\mathrm{O}_{\mathrm{t}}=\mathrm{V}_{\mathrm{k}}\right)=\mathcal{N}\left(\mathrm{V}_{\mathrm{k}}, \mu_{\mathrm{i}}, \sigma_{\mathrm{i}}\right)}_{\text {Emission probabilities matrix }}),
\end{gathered}
$$

where $\mu_{\mathrm{i}}$ and $\sigma_{\mathrm{i}}$ are the mean and variance of the distribution corresponding to the state $s_{\mathrm{i}}$ respectively, and $\mathcal{N}$ is Gaussian probability density function that can be defined as below:

$$
\mathrm{p}(\mathrm{x} \mid \mu, \sigma)=\mathcal{N}(\mathrm{x} \mid \mu, \sigma)=\frac{1}{\sqrt{2 \pi \sigma_{0}}} \exp \left(\frac{-(\mathrm{x}-\mu)^{2}}{2 \sigma}\right)
$$

Hence, the standard Gaussian HMM is specified by $\lambda=\{\mathrm{A}, \mu, \sigma, \pi\}$. 
TABLE 2 | Descriptive statistics of Diabetic and Non-diabetic population in our derived data sample.

\begin{tabular}{|c|c|c|c|}
\hline \multirow[t]{2}{*}{ Predictors } & \multicolumn{3}{|c|}{ Findings } \\
\hline & Total population & Progressors & Non-progressors \\
\hline & 1,918 & $584(23.49)$ & 1,334 \\
\hline \multicolumn{4}{|l|}{ Demographic (Gender, Age) } \\
\hline Male, sample size (\%) & $1143(38.96)$ & 280 & 495 \\
\hline Female, sample size (\%) & $775(61.03)$ & 304 & 839 \\
\hline Overall age mean \pm SD, Years & $63.19 \pm 11.89$ & $65.312 \pm 12.34$ & $58.937 \pm 14.315$ \\
\hline \multicolumn{4}{|l|}{ Vital Signs/clinical measures } \\
\hline Systolic BP, mean $\pm \mathrm{SD}, \mathrm{mm} \mathrm{Hg}$ & $128.611 \pm 15.86$ & $131.49 \pm 16.7$ & $128.496 \pm 17.259$ \\
\hline \multicolumn{4}{|l|}{ Lab Values } \\
\hline $\mathrm{FBS}, \mathrm{mmol} / \mathrm{L}$ mean $\pm \mathrm{SD}, \mathrm{mmol} / \mathrm{L}$ & $6.029 \pm 1.51$ & $7.256 \pm 2.056$ & $5.214 \pm 0.562$ \\
\hline Triglycerides, mean $\pm \mathrm{SD}, \mathrm{mmol} / \mathrm{L}$ & $1.72 \pm 1.02$ & $1.777 \pm 1.205$ & $1.419 \pm 0.837$ \\
\hline $\mathrm{HDL}$, sample size, mean $\pm \mathrm{SD}, \mathrm{mmol} / \mathrm{L}$ & $1.356 \pm 0.39$ & $1.249 \pm 0.361$ & $1.453 \pm 0.423$ \\
\hline $\mathrm{HbA}(1 \mathrm{c})$, mean $\pm \mathrm{SD}, \mathrm{mmol} / \mathrm{L}$ & $6.268 \pm 0.95$ & $6.821 \pm 1.049$ & $5.698 \pm 0.365$ \\
\hline Total Cholesterol mean $\pm \mathrm{SD}, \mathrm{mmol} / \mathrm{L}$ & $5.409 \pm 0.59$ & $4.433 \pm 1.224$ & $5.081 \pm 1.085$ \\
\hline $\mathrm{LDL}$, mean $\pm \mathrm{SD}, \mathrm{mmol} / \mathrm{L}$ & $2.442 \pm 0.851$ & $2.427 \pm 1.011$ & $3.007 \pm 0.939$ \\
\hline $\mathrm{BMl}$, mean $\pm \mathrm{SD}, \mathrm{kg} / \mathrm{m}^{2}$ & $29.81 \pm 6.362$ & $36.163 \pm 1197.454$ & $37.984 \pm 1697.225$ \\
\hline \multicolumn{4}{|l|}{ Depression frequency (\%) } \\
\hline YES & 373 & 112 & 261 \\
\hline NO & 1,544 & 472 & 1,073 \\
\hline \multicolumn{4}{|l|}{ Hypertension positive cases frequency (\%) } \\
\hline YES & 1,107 & 421 & 686 \\
\hline NO & 809 & 163 & 646 \\
\hline Unknown & 2 & & 2 \\
\hline
\end{tabular}

SD, standard deviation; BP, bnlood pressure; BMI, body mass index; FBS, fasting blood sugar; LDL, light density lipoprotein; HDL, high-density lipoprotein; HbA(1c), glycated hemoglobin.

Hold-out method was used in all modeling iterations to obtain an estimate of how well the model can generalize to an independent dataset. Thus, two subset of sequence of clinical measurements were considered, a training set and testing set. $80 \%$ of the data was used for training and the remaining $20 \%$ for testing.

Subsequently, for each of the 8 time horizons, Viterbi decoding method from HMM API (Hmmlearrn) was incorporated to train Gaussian HMM and carry out diagnostic and prognostic inferences related to diabetes risk in an individual over different time horizons. This is typically a Maximum a posteriori (MAP) estimation of the most likely sequence of hidden states, produced by the Viterbi algorithm given the observation sequence $\mathrm{O}=$ $\left\{\mathrm{O}_{\mathrm{t}}^{(\mathrm{l})}, \mathrm{t}=1,2, \ldots . \mathrm{T}, \mathrm{l}=1,2,3, \ldots \mathrm{L}\right\}$ and $\mathrm{O}_{\mathrm{t}} \in \mathrm{R}^{\mathrm{D}}$ where $\mathrm{T}$ is the length of each sequence and $l$ is the numbers of independent observation sequences, and model $\lambda=\{A, \mu, \sigma, \pi\}$. For further detail supplementary material is given in Presentation 1_v1.pdf.

After training the predictive model, the second task corresponds to the performance evaluation of Gaussian HMM at each of the 8 time points. Therefore, we estimated the discriminatory ability of each model via the Area under the Receiver Operating Characteristic Curve (AROC) computed over hold-out method. IBM SPSS Statistics (version 19) was used to perform statistical analysis in this study. Along with this the experiments used a combination of software tools developed in house and based on open source packages for Python (Version 2.7).

\section{RESULTS}

During 2003 to 2015, 172,168 individuals' of aged between 18 to 90 received healthcare services at CPCSSN, contributing over 8 million records, of these individuals, 40,317 individuals have diabetes, accounting for $23 \%$ of all cases morbidity during the study period. After applying inclusion criteria and approximation method, as mentioned above, to deal with irregularly sampled data the final dataset resulted in a total of 1918 individuals with 15,344 clinical visits recorded over 8-years. Approximately 584 (30.44\%) of individuals in our derived research sample were diabetic and among them $40.40 \%$ were women. Descriptive statistics of eligible cohort are reported in Table 2.

As a secondary analysis, we also performed a LR analysis to evaluate the significant $\mathrm{p}$-value of each risk factor included in our derived dataset in the context diabetes risk identification. According to the LR analysis except total cholesterol all the risk factors included in our research sample were statistically significant and added value to the model in prognosticating T2DM risk. HbA(1c) (Glycated Hemoglobin) was the most strongly associated with diabetes as compare to other risk factors included in this analysis, it remained the best predictor with odds ratios of $(\mathrm{p}<0.0005, \mathrm{OR}=12.565$ [95\% CI, 10.902 -14.482]). It demonstrated that $\mathrm{HbA}(1 \mathrm{c})$ solely was the prime risk factor with the ability to prognosticate the diabetes risk. Whereas FBG was ranked at second among the risk factors included in this study for prognosticating the diabetes risk ( $\mathrm{p}<$ 0.0005$, OR $=5.965[95 \% \mathrm{CI}, 5.607-1.281])$. To get a better understanding of what was going on inside the LR and to visualize the relative influence of each predictor for predicting diabetes risk we plotted Figure 1. It can also be observed that the B-value (-0.549) for LR equation for predicting the diabetes risk from the HDL is negative although it hold a significant $\mathrm{p}$-value (5.63E-24, 95\% CI). It demonstrated that increased level of HDL is associated with a reduced likelihood of diabetes onset ( $\mathrm{p}<$ $0.0005, \mathrm{OR}=.577$ [95\% CI, $0.480-0.695])$. However the B-values for the remaining risk factors are positive. 


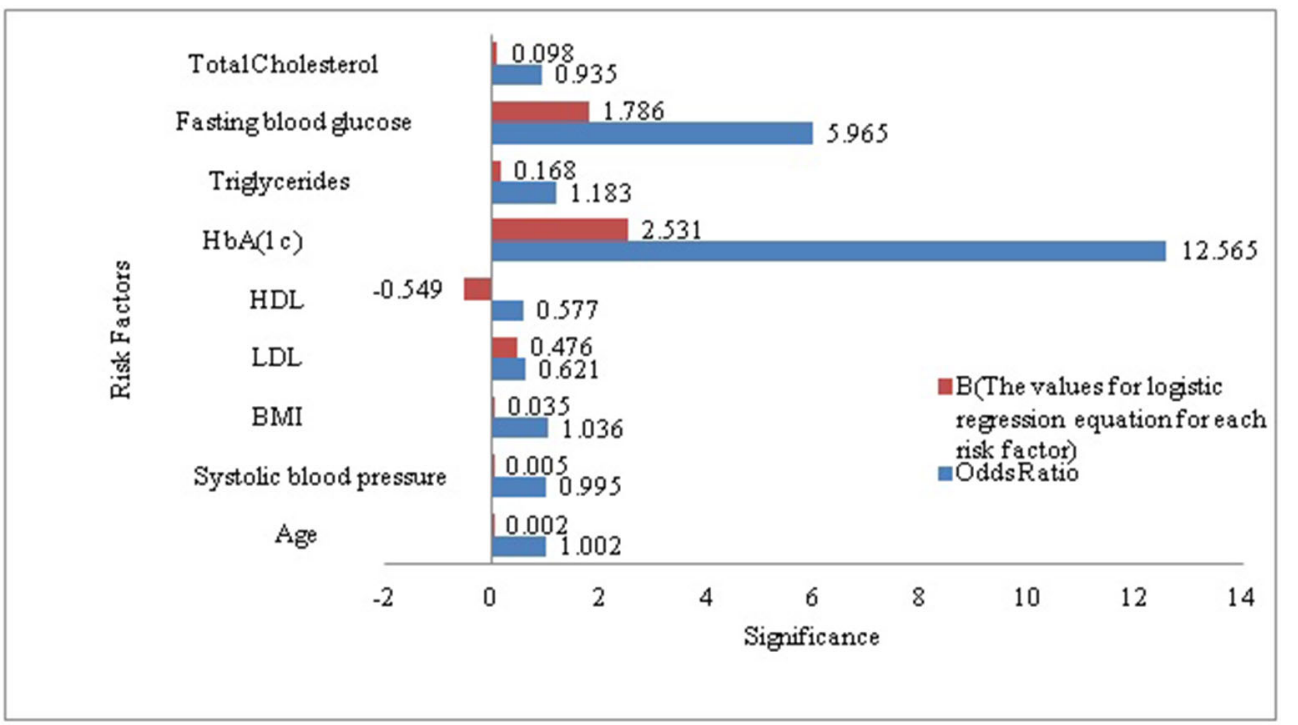

FIGURE 1 | Visualization of the association between diabetes and each of the risk factors included in our study sample.

As the objective of this proposed research is to prognosticate T2DM risk in an individual over different time horizon in order to make informed choices about future care and treatment with reduced complications and improved outcomes. Therefore, we prognosticate diabetes risk over a series of time horizon using only positively correlated and modifiable risk factors. Although this association provides some general guidance for diabetes but ineffective for individual risk assessment (Arbab-Zadeh and Fuster, 2015). In order to prove our proposed algorithm effectively, we make the contrast experiments. Figure 2 compares the predictive performance of our proposed method and standard HMM in term of AROCs for developing diabetes risk over different time horizon, using approximated and irregularly sampled data respectively. The AROC of our proposed method on our derived and approximated dataset was 0.81 ( $\mathrm{p}<0.0005$, [95\% CI, (0.791-0.847)]) for prediction window of 1 -year as compared to AROC 0.764(p $<0.0005$, [95\% CI, (0.741-0.794)]) with classical HMM without handling sparse and irregularly sampled multivariate time series data. It can be observed that the proposed method demonstrated significant performance over all the baseline models $(\mathrm{p}<0.0005)$ and time horizons. The highest AROC achieved (0.814) belonged to the 1year model with our proposed approach, as can be observed from Figure 2. Furthermore, experimental results also demonstrated that the AROC of our proposed model is consistently superior over all the time horizons as compare to baseline method. However, as expected, performance for both predictive models declines in relation to increasing time horizons. It can be observed that the predictive performance of our proposed model is at or above 0.795 AROC for prediction windows $\leq 4$ years whereas the performance is at 0.771 AROC for a 6-years prediction window. Performance then declines rapidly for prediction window lengths longer than 6 years.
According to the probabilistic prediction of HMM, we estimated 8 years risk of developing diabetes in our study sample, among 3 different risk categories with the cutoff value $<3,3$ to 9 and equal to 10 . We determined that $46 \%$ of individuals in our sample had a risk less than $3 \% ; 38 \%$ had a risk between $30 \%$ to $9 \%$ and $16 \%$ had a risk equal to $10 \%$.

\section{DISCUSSION}

The intensification in diabetes incidence is principal reason of increased diabetes prevalence. Early identification of individuals at high risk is imperative and a practical approach to prevent or delay the onset of diabetes through implementing proactive lifestyle and pharmacological interventions (Diabetes Prevention Program Research Group, 2003). In this context, physiological data contained in electronic medical records (EMRs) is the fundamental source for disease prognostic modeling (Tou et al., 2018). In addition, the rapid evolution in state of the art ML techniques offer a potentially promising means to accelerate discoveries, from EMRs data, which can be readily translated to clinical practice. From the clinicians' prospective, the development of such risk scoring techniques would allow them to allocate resources and healthcare services optimally and with more confidence (Vogenberg, 2009).

Hidden Markov Models and their variants have been widely deployed for modeling dynamical systems (Rabiner, 1989). These temporal latent variable models have also attained substantial success in various applications (Gruber et al., 2007; Fox et al., 2011). However, the format of the EMRs data together with the nature of the clinical setting poses various significant challenges that confound standard HMM. In particular, the dynamic range of the time scale in EMRs is one of the potentially contributing 


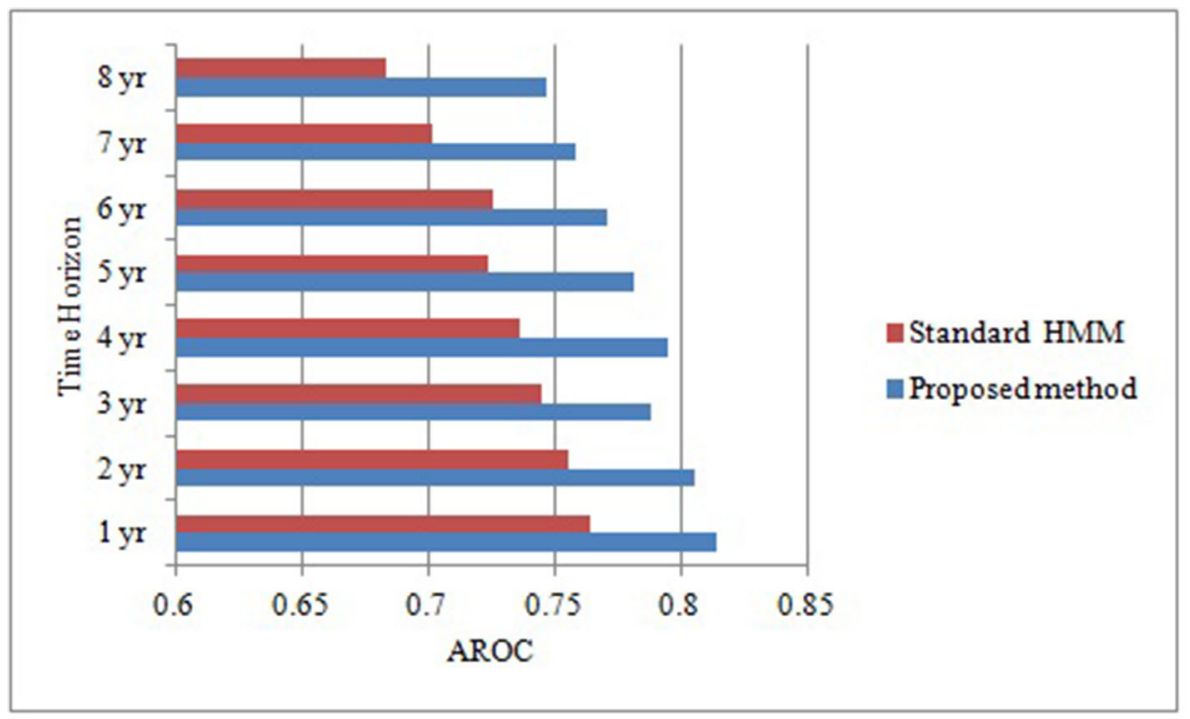

FIGURE 2 | Comparative analysis of area under receiver operating characteristic curve of our proposed method and standard HMM over different time horizons

factor for sparse and irregularly sampled clinical data. Typically the HMM presumes that the training data sample is collected regularly at discrete time intervals. Thus, direct incorporation of EMRs with observations sequence irregularly or sparsely sampled into standard HMM [(e.g. the models in Fox et al. (2011) and Rabiner (1989)] will not suffice for jointly describing the latent states and hence ensuring accurate inferences. This paper presented a new hybrid approach combining approximation technique as a component with Hidden Markov Model (HMM) in order to deal with sparse and irregularly sampled time series data for effectively determining the risk of developing diabetes in an individual over different time horizons. The proposed method is fully modular. It basically incorporated an approximation method based on NDDM to handle multivariate sparse and irregularly sampled data as dynamical systems inputs, followed by the application of HMM based diagnostic predictive model that operates over the regularly spaced time series output provided by the approximation method.

In order to develop the prognostic prediction model we incorporated further two step approach. Therefore, we also incorporated LR analysis in order to identify potentially contributing risk factors of diabetes. In LR analysis we considered 0.05 level of significance, as depicted in Table 3.

Table 3 shows the results considering a significant level of 0.05. It depicts highly significant association between each risk factor and diabetes expect total cholesterol. This means that all the risk factors added value to the model for diabetes onset prediction excluding total cholesterol. As the total cholesterol exhibited negative association with the T2DM thus we excluded it from the contributing risk factors. Along with this, we also excluded gender from the potentially contributing risk factors. As gender is a non modifiable risk factor; thus, provide not much guidance for diabetes prevention. Wilson et al. (2007) also not recommended gender as a candidate risk factor for the prediction of diabetes onset. On the other hand age is also a non modifiable risk factor but we did not exclude it from contributing risk factors because several existing research conducted by Stern et al. (2002), Lindstrom et al. (2003), Kanaya et al. (2005), Zhou et al. (2013) and Perveen et al. (2016) suggested that age is a potentially significant risk factor for prognostic prediction of diabetes risk. These studies also highlighted that elderly population had higher risk for developing T2DM than those with younger age. In addition, our results also demonstrated that age is a significant influencing factor for diabetes $(\mathrm{p}<0.0005$, OR $=1.002[95 \%$ CI, $0.999-1.006]$ ) as depicted in Table 3.

Nevertheless, HDL is statistically significant risk factor ( $\mathrm{p}<$ $0.0005, \mathrm{OR}=.577$ [95\% CI, $0.480-0.695]$ ) but have a negative association with the development of diabetes risk in an individual (Mazzone et al., 2006; Lincoff et al., 2007). According to our analysis the probability of developing diabetes reduced approximately $5 \%$ with one unit increase in HDL level.While increased levels of FBS and $\mathrm{HbA}(1 \mathrm{c})$ in blood is strongly associated with higher risk of developing T2DM. It can also be observed from the concise adds ratios that $\mathrm{HbA}(1 \mathrm{c})$ is the strongest predictor for prognosticating diabetes risk. Furthermore, Statistical analysis results of our proposed study are also commensurate with the results of the existing research proposed by Mackey et al. (2015), Wang et al. (2016) and Perveen et al. (2018a).

To evaluate the impact of modeling sparse and irregular time series data using approximation technique based on NDDM we conducted a set of experiments on both the original and approximated synthetic dataset. In both cases the baseline model is GausianHMM however the input to one of them is sparse and irregularly sampled time series. The output is a single scalar representing the predicted class along with the probability distribution over a set of class values. 
TABLE 3 | Analysis of the association between individual risk factor and diabetes risk in our study sample.

\begin{tabular}{lcc}
\hline Explanatory variables & OR $(\mathbf{9 5} \% \mathbf{C . I . )}$ & P Value \\
\hline Age & $1.002(.999-1.006)$ & $3.49 \mathrm{E}-22$ \\
Systolic blood pressure & $.995(.993-.997)$ & $7.02 \mathrm{E}-07$ \\
$\mathrm{BMl}$ & $1.036(1.0301-.042)$ & $1.60 \mathrm{E}-58$ \\
$\mathrm{LDL}$ & $.621(.528-.732)$ & $1.56 \mathrm{E}-23$ \\
$\mathrm{HDL}$ & $.577(.480-.695)$ & $5.63 \mathrm{E}-24$ \\
$\mathrm{HbA}(1 \mathrm{c})$ & $12.565(10.902-14.482)$ & $7.94 \mathrm{E}-143$ \\
Triglycerides & $1.183(1.093-1.281)$ & $5.34 \mathrm{E}-07$ \\
Fasting blood glucose & $5.965(5.607-1.281)$ & 0.000 \\
Total Cholesterol & $.935(.795-1.098)$ & 0.411 \\
Intercept & & $3.63 \mathrm{E}-145$
\end{tabular}

Nagelkerke $R 2=0.546$.

Hosmer and Lemeshow Test $=0.360$ (Significantly greater than 0.0005).

$\mathrm{OR}$, odds ratio; C.I., confidence interval; $S B P$, systolic blood pressure; BMI, body mass index; $L D L$, light density lipoprotein; $H D L$, high density lipoprotein; $H b A(1 c)$, glycated hemoglobin.

Validation of the accuracy of a prognostic prediction model is often involves plotting observed incidents verses estimated probability to observe visually how close model predictions were to actual predictions (Buijsse et al., 2011). Therefore, we also incorporated AROC to evaluate the discriminatory capability of our proposed model in identify 8 -years risk of diabetes.

In the results, the reported AROC for the proposed method on our derived dataset was 0.81 ( $\mathrm{p}<0.0005$, [95\% CI, (0.791$0.847)$ ]) for prediction window of 1-year, showing a high discriminative capability as compared to standard HMM with AROC 0.764 ( $p<0.0005$, [95\% CI, (0.741-0.794)]). It can also be concluded that dealing with sparse and irregularly sampled multivariate time series data can yield relatively better performance. Furthermore, experimental results also demonstrated that the AROC of our proposed model is consistently superior over all the time horizons as compare to standard HMM learned, indicating that the model learned using approximation approach based on NDDM have the potential to discriminate persons who will have the diabetes from those who did not with considerably high performance.

In addition to identifying diabetes risk a-prior, this is the first study that proposed an approximation technique based on NDDM to deal with the sparse and irregularly sampled EMR data before developing prognostic model. Furthermore, the proposed method has the ability to effectively estimate the future risk of T2DM with reduced healthcare expenditures.

The total estimated direct and indirect healthcare expenditures for diabetic patients were $\$ 13,700$ per year, whereas about $\$ 7,900$ of this amount was particularly attributed to diabetes (American Diabetes Association, 2013). It is also estimated that diabetic patients have healthcare expenditures, on average, 2.3 times higher than what expenditures would be in the absence of diabetes. As the predictive performance of our proposed method was comparatively good, therefore, we also estimated the 8-years risk of developing T2DM among 1458 non-diabetic individuals for whom data was available in 2015. According to the probabilistic results of our proposed method over the baseline data set it can be observed that approximately $15.8 \%(231)$ individuals have significant risk of developing T2DM in the next 8-years interval ranging from 2015 to 2022. Given the newly identified individuals with increased risk of developing T2DM, we can save a considerable fraction of individuals from our baseline data set if healthcare providers promptly manage those vulnerable.

There are some remarkable advances and benefits of the proposed research. In this study we performed prognostic prediction of diabetes risk over a set of time horizons using EMRs data collected for secondary purposes not for research. Therefore it is a time and cost effective approach. According to $\mathrm{LR}$ analysis it can also be concluded that $\mathrm{HbA}(1 \mathrm{c})$ is the strongest predictor for prognosticating diabetes risk and has the ability to solely predict it. As fasting is not prerequisite for The $\mathrm{HbA}(1 \mathrm{c})$ test and can be conducted at any time of the day. Therefore, it can easily be used in community screening programs or an opportunistic screening in outpatient visits. Furthermore, in our derived dataset each laboratory test (i.e. FBG, LDL, HbA1c, HDL and triglycerides) was measured and recorded for each time horizon in CPCSSN. However, a limitation of the study is that the proposed model was only validated internally caution is required when applied over other populations in order to minimize the bias in generalization.

\section{CONCLUSION}

In summary, our results demonstrated that the proposed method has the capability to deal with sparse and irregularly sampled data for leveraging EMRs to learn underlying hidden state with the objective to provide insights into the disease process over a series of time horizon. Furthermore, the proposed method conceal the inherent temporal dependencies exist in the temporal data, required for decisive step to characterize disease risk in an individual with significantly improved predictive performance as compare to standard HMM. Therefore, this is an encouraging step forward for active identification of high risk individuals as a means to propel healthcare toward an innovative preventive orientation for diabetes. In future further research is warranted for the cost effective analysis of the proposed study. This can be extended to prognosticate the future risk of other type of ailments particularly chronic diseases.

\section{DATA AVAILABILITY STATEMENT}

The datasets generated for this study are available on request to the corresponding author.

\section{ETHICS STATEMENT}

The studies involving human participants were reviewed and approved by Ryerson University research ethics board, Ryerson University, Toronto, Ontario, Canada. The patients/participants provided their written informed consent to participate in this study. 


\section{AUTHOR CONTRIBUTIONS}

All authors contributed equally to the conception, design, and development of the research. SP investigated/predicted the risk of developing type 2 diabetes in an individual using EMRs data. MS provided the technical guidance for conducting the research and analysis of the data. KK critically revised the paper draft for the soundness of the research from the medical viewpoint. AG critically revised the paper draft for the soundness of the research from the machine learning viewpoint. All authors reviewed the manuscript before its submission. MA also provided the technical guidance for conducting the research.

\section{REFERENCES}

Alberti, K. G. M. M., and Zimmet, P. F. (1998). Definition, diagnosis and classification of diabetes mellitus and its complications. Part 1: diagnosis and classification of diabetes mellitus. Provisional report of a WHO consultation. Diabetic Med. 15 (7), 539-553. doi: 10.1002/(SICI)1096-9136(199807)15:7< 539::AID-DIA668>3.0.CO;2-S

American Diabetes Association (2013). Economic costs of diabetes in the US in 2012. Diabetes Care 36, 1033-1046. doi: 10.2337/dc12-2625

American Diabetes Association. (2014). Diagnosis and classification of diabetes mellitus. Diabetes Care 37 (Supplement 1), S81-S90. doi: 10.2337/ dc14-S081

Arbab-Zadeh, A., and Fuster, V. (2015). The myth of the "vulnerable plaque": transitioning from a focus on individual lesions to atherosclerotic disease burden for coronary artery disease risk assessment. J. Am. Coll. Cardiol. 65 (8), 846-855. doi: 10.1016/j.jacc.2014.11.041

Artières, T., Marchand, J. M., Gallinari, P., and Dorizzi, B. (2000). "Stroke level modeling of on line handwriting through multi-modal segmental models," in International Workshop on Frontiers in Handwriting Recognition (IWFHR). (Amsterdam).

Atlas, D. (2015). "International diabetes federation," in IDF Diabetes Atlas, 7th edn (Brussels, Belgium: International Diabetes Federation). doi: 10.1214/aoms/ 1177699147

Baum, L. E., and Petrie, T. (1966). Statistical inference for probabilistic functions of finite state Markov chains. Ann. Math. Stat 37 (6), 1554-1563.

Birkhead, G. S., Klompas, M., and Shah, N. R. (2015). Uses of electronic health records for public health surveillance to advance public health. Annu. Rev. Public Health 36, 345-359. doi: 10.1146/annurev-publhealth -031914-122747

Buijsse, B., Simmons, R. K., Griffin, S. J., and Schulze, M. B. (2011). Risk assessment tools for identifying individuals at risk of developing type 2 diabetes. Epidemiol. Rev. 33 (1), 46-62. doi: 10.1093/epirev/mxq019

Chen, L., Magliano, D. J., Balkau, B., Colagiuri, S., Zimmet, P. Z., Tonkin, A. M., et al. (2010). AUSDRISK: an Australian Type 2 Diabetes Risk Assessment Tool based on demographic, lifestyle and simple anthropometric measures. Med. J. Aust. 192 (4), 197-202. doi: 10.5694/j.1326-5377.2010.tb03478.x

Diabetes Prevention Program Research Group (2003). Costs associated with the primary prevention of type 2 diabetes mellitus in the diabetes prevention program. Diabetes Care 26 (1), 36-47. doi: 10.2337/diacare.26.1.36

Einarson, T. R., Acs, A., Ludwig, C., and Panton, U. H. (2018). Prevalence of cardiovascular disease in T2DM: a systematic literature review of scientific evidence from across the world in 2007-2017. Cardiovasc. Diabetol. 17 (1), 83. doi: 10.1186/s12933-018-0728-6

El Nahas, M., Kassim, S., and Shikoun, N. (2012). Profile hidden markov model for detection and prediction of hepatitis $\mathrm{C}$ virus mutation. Int. J. Comput. Sci. Issues (IJCSI) 9 (5), 251.

Emerging Risk Factors Collaboration (2010). Diabetes mellitus, fasting blood glucose concentration, and risk of vascular disease: a collaborative metaanalysis of 102 prospective studies. Lancet 375 (9733), 2215-2222. doi: 10.1016/S0140-6736(10)60484-9

Fox, E. B., Sudderth, E. B., Jordan, M. I., and Willsky, A. S. (2011). A sticky HDPHMM with application to speaker diarization. Ann. Appl. Stat 5 (2A), $1020-$ 1056. doi: 10.1214/10-AOAS395

\section{ACKNOWLEDGMENTS}

The authors would like to thank CPCSSN for their support on the EMRs databases, and NSERC for partial funding of this research.

\section{SUPPLEMENTARY MATERIAL}

The Supplementary Material for this article can be found online at: https://www.frontiersin.org/articles/10.3389/fgene. 2019.01076/full\#supplementary-material

Gregg, E. W., Li, Y., Wang, J., Rios Burrows, N., Ali, M. K., Rolka, D., et al. (2014). Changes in diabetes-related complications in the United States, 1990-2010. New Engl. J. Med. 370 (16), 1514-1523. doi: 10.1056/NEJMoa1310799

Gruber, A., Weiss, Y., and Rosen-Zvi, M. (2007). "Hidden topic markov models," in Artificial intelligence and statistics. (San Juan, Puerto Rico), 163-170.

Gunter, T. D., and Terry, N. P. (2005). The emergence of national electronic health record architectures in the United States and Australia: models, costs, and questions. J. Med. Internet Res. 7 (1), e3. doi: 10.2196/jmir.7.1.e3

Hoiles, W., and Van Der Schaar, M. (2016). "A non-parametric learning method for confidently estimating patient's clinical state and dynamics," in Advances in Neural Information Processing Systems. (UK), 2020-2028.

Kahn, H. S., Cheng, Y. J., Thompson, T. J., Imperatore, G., and Gregg, E. W. (2009). Two risk-scoring systems for predicting incident diabetes mellitus in US adults age 45 to 64 years. Ann. Internal Med. 150 (11), 741-751. doi: 10.7326/0003-4819-150-11-200906020-00002

Kalu, E. E. (2009). Numerical Methods with Applications: Abridged. Lulu.com.

Kanaya, A. M., Fyr, C. L. W., De Rekeneire, N., Shorr, R. I., Schwartz, A. V., Goodpaster, B. H., et al. (2005). Predicting the development of diabetes in older adults: the derivation and validation of a prediction rule. Diabetes Care 28 (2), 404-408. doi: 10.2337/diacare.28.2.404

Kenny, P., Lennig, M., and Mermelstein, P. (1990). Speaker adaptation in a largevocabulary Gaussian HMM recognizer. IEEE Trans. Pattern Anal. Mach. Intell. 12 (9), 917-920. doi: 10.1109/34.57686

Knowler, W. C., Barrett-Connor, E., Fowler, S. E., Hamman, R. F., Lachin, J. M., Walker, E. A., et al. (2002). Reduction in the incidence of type 2 diabetes with lifestyle intervention or metformin. New Engl. J. Med. 346 (6), 393-403. doi: 10.1056/NEJMoa012512

Lai, K., Twine, N., O’brien, A., Guo, Y., and Bauer, D. (2016). Artificial Intelligence and Machine Learning in Bioinformatics.

Li, S. C. X., and Marlin, B. M. (2016). "A scalable end-to-end gaussian process adapter for irregularly sampled time series classification," in Advances in neural information processing systems. (UK), 1804-1812.

Li, G., Zhang, P., Wang, J., Gregg, E. W., Yang, W., Gong, Q., et al. (2008). The long-term effect of lifestyle interventions to prevent diabetes in the China Da Qing Diabetes Prevention Study: a 20-year follow-up study. Lancet 371 (9626), 1783-1789. doi: 10.1016/S0140-6736(08)60766-7

Liebl, A., Goertz, A., Henkel, B., and Spannheimer, A. (2000). "Costly type 2 diabetes mellitus. Does diabetes cost 20 billion per year? MMW Fortschr. Med. 142 (23), 39-42

Lincoff, A. M., Wolski, K., Nicholls, S. J., and Nissen, S. E. (2007). Pioglitazone and risk of cardiovascular events in patients with type 2 diabetes mellitus: a metaanalysis of randomized trials. Jama 298 (10), 1180-1188. doi: 10.1001/ jama.298.10.1180

Lindström, J., and Tuomilehto, J. (2003). The diabetes risk score: a practical tool to predict type 2 diabetes risk. Diabetes Care 26 (3), 725-731. doi: 10.2337/ diacare.26.3.725

Lindstrom, J., Louheranta, A., Mannelin, M., and Rastas, M. (2003). The Finnish Diabetes Prevention Study (DPS): Lifestyle intervention and 3-year results on diet and physical activity. Diabetes Care 26 (12), 3230. doi: 10.2337/diacare.26.12.3230

Liu, Y. Y., Li, S., Li, F., Song, L., and Rehg, J. M. (2015). "Efficient learning of continuous-time hidden markov models for disease progression," in Advances in neural information processing systems. (UK), 3600-3608. 
Mackey, R. H., Mora, S., Bertoni, A. G., Wassel, C. L., Carnethon, M. R., Sibley, C. T., et al. (2015). Lipoprotein particles and incident type 2 diabetes in the multiethnic study of atherosclerosis. Diabetes Care 38 (4), 628-636. doi: 10.2337/ dc14-0645

Mashayekhi, M., Prescod, F., Shah, B., Dong, L., Keshavjee, K., and Guergachi, A. (2015). Evaluating the performance of the Framingham Diabetes Risk Scoring Model in Canadian electronic medical records. Can. J. Diabetes 39 (2), 152156. doi: $10.1016 /$ j.jcjd.2014.10.006

Mathews, J. H. (1986). Numerical methods for computer science, engineering, and mathematics (Prentice-Hall, Inc).

Mazzone, T., Meyer, P. M., Feinstein, S. B., Davidson, M. H., Kondos, G. T., D'Agostino, R. B., et al. (2006). Effect of pioglitazone compared with glimepiride on carotid intima-media thickness in type 2 diabetes: a randomized trial. Jama 296 (21), 2572-2581. doi: 10.1001/jama.296.21.joc60158

McEwen, L. N., and Herman, W. H. (2017). "Health care utilization and costs of diabetes," in Diabetes in America, 3rd ed. Eds. C. C. Cowie, S. S. Casagrande and A. Menke (Bethesda, MD: National Institutes of Health).

Pambianco, G., Costacou, T., Ellis, D., Becker, D. J., Klein, R., and Orchard, T. J. (2006). The 30-year natural history of type 1 diabetes complications: the Pittsburgh Epidemiology of Diabetes Complications Study experience. Diabetes 55 (5), 1463-1469. doi: 10.2337/db05-1423

Perveen, S., Shahbaz, M., Guergachi, A., and Keshavjee, K. (2016). Performance analysis of data mining classification techniques to predict diabetes. Proc. Comput. Sci. 82, 115-121. doi: 10.1016/j.procs.2016.04.016

Perveen, S., Shahbaz, M., Keshavjee, K., and Guergachi, A. (2018a). Metabolic Syndrome and Development of Diabetes Mellitus: Predictive Modeling Based on Machine Learning Techniques. IEEE Access 7, 1365-1375. doi: 10.1109/ ACCESS.2018.2884249

Perveen, S., Shahbaz, M., Keshavjee, K., and Guergachi, A. (2018b). A systematic machine learning based approach for the diagnosis of non-alcoholic fatty liver disease risk and progression. Sci. Rep. 8 (1), 2112. doi: 10.1038/s41598-01820166-x

Rabiner, L. R. (1989). A tutorial on hidden Markov models and selected applications in speech recognition. Proc. IEEE 77 (2), 257-286. doi: 10.1109/5.18626

Ramachandran, A. (2014). Know the signs and symptoms of diabetes. Indian J. Med. Res. 140 (5), 579.

Schulam, P., and Saria, S. (2015). "A framework for individualizing predictions of disease trajectories by exploiting multi-resolution structure," in Advances in Neural Information Processing Systems. (UK), 748-756.

Srikanth, P. (2015). Using Markov chains to predict the natural progression of diabetic retinopathy. Int. J. Ophthalmol. 8 (1), 132.
Stern, M. P., Williams, K., and Haffner, S. M. (2002). Identification of persons at high risk for type 2 diabetes mellitus: do we need the oral glucose tolerance test? Ann. Internal Med. 136 (8), 575-581. doi: 10.7326/0003-4819-136-8-200204160-00006

Tou, H., Yao, L., Wei, Z., Zhuang, X., and Zhang, B. (2018). Automatic infection detection based on electronic medical records. BMC Bioinf. 19 (5), 117. doi: 10.1186/s12859-018-2101-x

Vogenberg, F. R. (2009). Predictive and prognostic models: implications for healthcare decision-making in a modern recession. Am. Health Drug Benefits 2 (6), 218.

Wang, A., Chen, G., Su, Z., Liu, X., Liu, X., Li, H., et al. (2016). Risk scores for predicting incidence of type 2 diabetes in the Chinese population: the Kailuan prospective study. Sci. Rep. 6, 26548. doi: 10.1038/srep26548

Wild, S., Roglic, G., Green, A., Sicree, R., and King, H. (2004). Global prevalence of diabetes: estimates for the year 2000 and projections for 2030. Diabetes Care 27 (5), 1047-1053. doi: 10.2337/diacare.27.5.1047

Wilson, P. W., Meigs, J. B., Sullivan, L., Fox, C. S., Nathan, D. M., and D'Agostino, R. B. (2007). Prediction of incident diabetes mellitus in middle-aged adults: the Framingham Offspring Study. Arch. Internal Med. 167 (10), 1068-1074. doi: 10.1001/archinte.167.10.1068

Yoon, J., Alaa, A., Hu, S., and Schaar, M. (2016). "ForecastICU: a prognostic decision support system for timely prediction of intensive care unit admission," in International Conference on Machine Learning. (Vienna, Austria), 1680-1689.

Zhou, X., Qiao, Q., Ji, L., Ning, F., Yang, W., Weng, J., et al. (2013). Nonlaboratorybased risk assessment algorithm for undiagnosed type 2 diabetes developed on a nation-wide diabetes survey. Diabetes Care 36 (12), 3944-3952. doi: 10.2337/ dc13-0593

Zou, Q., Qu, K., Luo, Y., Yin, D., Ju, Y., and Tang, H. (2018). Predicting diabetes mellitus with machine learning techniques. Front. Genet. 9, 515. doi: 10.3389/ fgene.2018.00515

Conflict of Interest: The authors declare that the research was conducted in the absence of any commercial or financial relationships that could be construed as a potential conflict of interest.

Copyright (c) 2020 Perveen, Shahbaz, Ansari, Keshavjee and Guergachi. This is an open-access article distributed under the terms of the Creative Commons Attribution License (CC BY). The use, distribution or reproduction in other forums is permitted, provided the original author(s) and the copyright owner(s) are credited and that the original publication in this journal is cited, in accordance with accepted academic practice. No use, distribution or reproduction is permitted which does not comply with these terms. 